\title{
INTEGRATING THE HEALTHCARE ENTERPRISE IN RADIATION ONCOLOGY PLUG AND PLAY - THE FUTURE OF RADIATION ONCOLOGY?
}

\author{
May Abdel-Wahab, M.D., Ph.D., ${ }^{*}$ Ramesh Rengan, M.D., Ph.D., ${ }^{\dagger}$ Bruce Curran, M.S., M.E., ${ }^{\ddagger}$ \\ Stuart Swerdloff, Ph.D. ${ }^{\S}$ Mika Miettinen, ${ }^{ף}$ Colin Field, M.Sc., ${ }^{* *}$ Sunita RanjitKar, M.S.H.S. ${ }^{\dagger \dagger}$ \\ Jatinder Palta, Ph.D. ${ }^{\S \S}$ and Prabhakar Tripuraneni, M.D. ${ }^{\text {ฯ }}$ \\ *Department of Radiation Oncology, Miller School of Medicine, University of Miami, Miami, Florida; ${ }^{\dagger}$ Department of Radiation \\ Oncology, University of Pennsylvania, Philadelphia, Pennsylvania; ${ }^{\ddagger}$ Department of Radiation Oncology, Rhode Island Hospital, Brown \\ University, City, Rhode Island; ${ }^{\complement}$ Elekta, Sunnyvale , California; ${ }^{\uparrow}$ Varian Systems, Palo Alto, California; **Cross Cancer Institute, \\ Edmonton, Alberta, Canada; ${ }^{\dagger \dagger}$ American Society for Therapeutic Radiology and Oncology, Fairfax, Virginia ${ }^{\S \S}$ Department of Radiation \\ Oncology, University of Florida, Gainesville, Florida and ${ }^{\top \uparrow}$ Department of Radiation Oncology, Scripps Institute, La Jolla, California
}

\begin{abstract}
Purpose: To describe the processes and benefits of the integrating healthcare enterprises in radiation oncology (IHE-RO).

Methods: The IHE-RO process includes five basic steps. The first step is to identify common interoperability issues encountered in radiation treatment planning and the delivery process. IHE-RO committees partner with vendors to develop solutions (integration profiles) to interoperability problems. The broad application of these integration profiles across a variety of vender platforms is tested annually at the Connectathon event. Demonstration of the seamless integration and transfer of patient data to the potential users are then presented by vendors at the public demonstration event. Users can then integrate these profiles into requests for proposals and vendor contracts by institutions.

Results: Incorporation of completed integration profiles into requests for proposals can be done when purchasing new equipment. Vendors can publish IHE integration statements to document the integration profiles supported by their products. As a result, users can reference integration profiles in requests for proposals, simplifying the systems acquisition process. These IHE-RO solutions are now available in many of the commercial radiation oncology-related treatment planning, delivery, and information systems. They are also implemented at cancer care sites around the world.
\end{abstract}

Conclusions: IHE-RO serves an important purpose for the radiation oncology community at large. (C) 2010 Elsevier Inc.

Radiation oncology, Connectivity, IHE, IHE-RO, Radiation Planning.

\section{INTRODUCTION}

Radiation oncology is a dynamic and rapidly evolving field using cutting edge advances in radiation delivery and advanced imaging. The past decade has seen significant advances in both of these spheres. For radiation oncologists to incorporate these advances into treatment design and delivery for their patients, a seamless integration and flow of imaging data into radiation treatment planning are required. Furthermore, once the treatment planning process is initiated, patient data sets must flow seamlessly from the CT simulator to dosimetry to the linear accelerator for delivery to the patient; and once the treatment has been initiated, imaging and dosimetric data must be collected to confirm, record, and verify accuracy of delivery, and allow for modifications when necessary.
All too often, however, these data transfers are far from seamless, and transfer is more analogous to an obstacle course, where data integrity could be compromised at each transition. A simple example that occurs almost daily in routine radiation oncology practice is the problem of incorporating imaging data, such as positron emission tomography/ computed tomography $(\mathrm{CT})$ or magnetic resonance imaging obtained at outside institutions, into treatment planning and delivery. Some of the issues and limitations of communication between various radiation oncology systems and within each system were discussed by Swerdloff (1) and may potentially limit the seamless development of more advanced planning and treatment systems in the future. Furthermore, the differences between various planning and/or treatment systems, as well as the purchase of different systems by different

Conflict of interest: none.

Received Sept 30, 2009. Accepted for publication Oct 1, 2009.
Reprint requests to: May Abdel-Wahab, M.D., Department of Radiation Oncology, Miller School of Medicine, University of Miami, 1475 NW 12th Ave. (D-31), Miami, Fl 33136. Tel: (305) 243-4210; Fax: (305) 243-4363; E-mail: mwahab@med.miami.edu 
departments, limit the exchange of patient information and may adversely affect patient care and the timely intervention or treatment due to technical factors related to interoperability. Integrating the Healthcare Enterprise (IHE) began in 1998 under the leadership of the Healthcare Information Management Systems Society (HIMMS) and the Radiological Society of North America (RSNA) as an effort to establish standards by which various pieces of medical equipment can communicate. In 2004, the American Society for Radiation Oncology (ASTRO) formed a multisociety, multinational, multispecialty task force, Integrating the Healthcare Enterprise-Radiation Oncology (IHE-RO), to address issues of interoperability and information sharing that impact the quality of care in radiation oncology. The increasing recognition of these issues facing optimal communication within the healthcare system and similar impediments to optimal patient care led to the IHE effort. This effort will also have an impact on the collection, interpretation, and sharing of research protocol information.

\section{Mission of IHE-RO}

The mission of the IHE-RO task force is to promote seamless connectivity and integration of radiotherapy equipment and patient health information systems. The task force includes members from ASTRO, RSNA, American Association of Physicists in Medicine (AAPM), National cancer Institute (NCI/NIH), the American College of Radiology (ACR), and the National Electrical Manufacturers Association (NEMA) and more than 20 academic institutions and cancer centers. In addition, members of the international community invited to participate include the Japanese Society for Therapeutic Radiology and Oncology (JASTRO), Latin American Society of Therapeutic Radiation Oncology (ALATRO), Egyptian Cancer Society/Egyptian National Cancer Institute (ECS), Chinese Society of Radiation Oncology, European Society for Therapeutic Radiology and Oncology (ESTRO) and the International Atomic Energy Agency (IAEA). The large, diverse list of vendors participating in this effort includes Varian Systems, Impac, Elekta, Philips, BrainLAB, Nucletron, Siemens, Accuray, Tomotherapy, Calypso, Cogent health solutions, CMS, Fuji Medical systems, Kodak Health group, ATT global TSG integrations, Sun Nuclear, Resonant Medical, Dynamic Imaging, Navotek, Borardus Medical, IBA particle therapy, VisionRT, and many more. The IHE-RO task force, in close collaboration with radiotherapy product manufacturers, has been developing and will continue to develop appropriate integration profiles for radiation therapy and has set up a demonstration of seamless communication among the full array of radiotherapy products.

\section{METHODS}

\section{Structure of IHE-RO}

The IHE-RO task force consists of a planning committee and a technical committee. The membership of these committees along with schedules of activities, meeting agendas, minutes, and working documents can be found at the IHE Wiki website (http://wiki.ihe.net).

1. Planning committee. The planning committee includes physicists, physicians, and vendor representatives. The committee identifies interoperability problems and develops examples of hypothetical clinical scenarios where software compatibility issues stand in the way of clinical care (use cases).

2. Technical committee. The technical committee, which includes physicists and vendor representatives, studies the cases submitted by the planning committee and determines the technical requirements and feasibility of solutions to the issues raised in the use case. The technical committee formulates a solution to the issues and creates a document describing the technical aspects of the solution, known as the "integration profile," examples of which include normal treatment planning, multimodality registration for radiation oncology, and radiation therapy (RT) treatment workflow. Vendors then are able to use the profile as an implementation guide in developing applications that meet the requirements as written. Their implementations are then tested using "test tools" developed under contract for the IHE-RO effort.

\section{Integration profiles: the IHE process}

The technical and planning committees work together to complete the different phases of the IHE process as described below. The overarching process can be divided into five basic steps: (I) identify common interoperability issues encountered in the radiation treatment planning and delivery process, (2) partner with vendors to develop solutions (also referred to as integration profiles) to the interoperability problems, (3) test the broad application of these integration profiles across a variety of vender platforms at the "Connectathon" event, (4) facilitate demonstration of the application by vendors of these integration profiles and how they facilitate seamless integration and transfer of patient data to the potential users at the "public demonstration" event, and (5) publish integration profiles to allow users to integrate these into requests for proposals and vendor contracts by institutions.

a. Identifying interoperability problems. The planning committee organizes the definition of "use cases." Use cases are basically common radiation oncology integration problems that may be related to access to information, clinical workflow, administration, and underlying infrastructure. These use cases are submitted to the technical committee which then determines the technical requirements and feasibility of solving the use case and then establish priorities that determine which use cases can be concurrently solved.

$b$. Development of integration profiles. The technical committee selects standards that address each use case identified. The committee then creates a document describing the proposed IHE integration profile. Integration profiles or solutions that have already

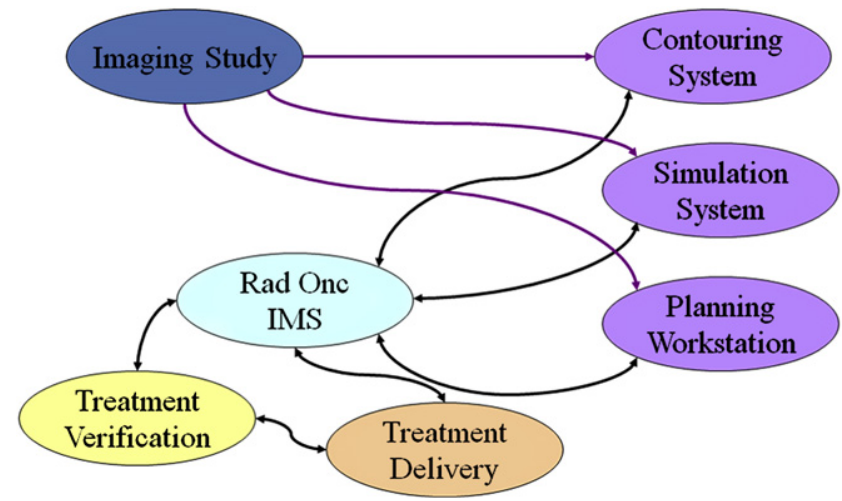

Fig. 1. Basic interoperability, image registration, and multimodality. (Courtesy of Bruce Curran, Dept. of Radiation Oncology, Rhode Island Hospital, Brown University, City, Rhode Island). Rad One IMS = Radiation oncology Information management systems 


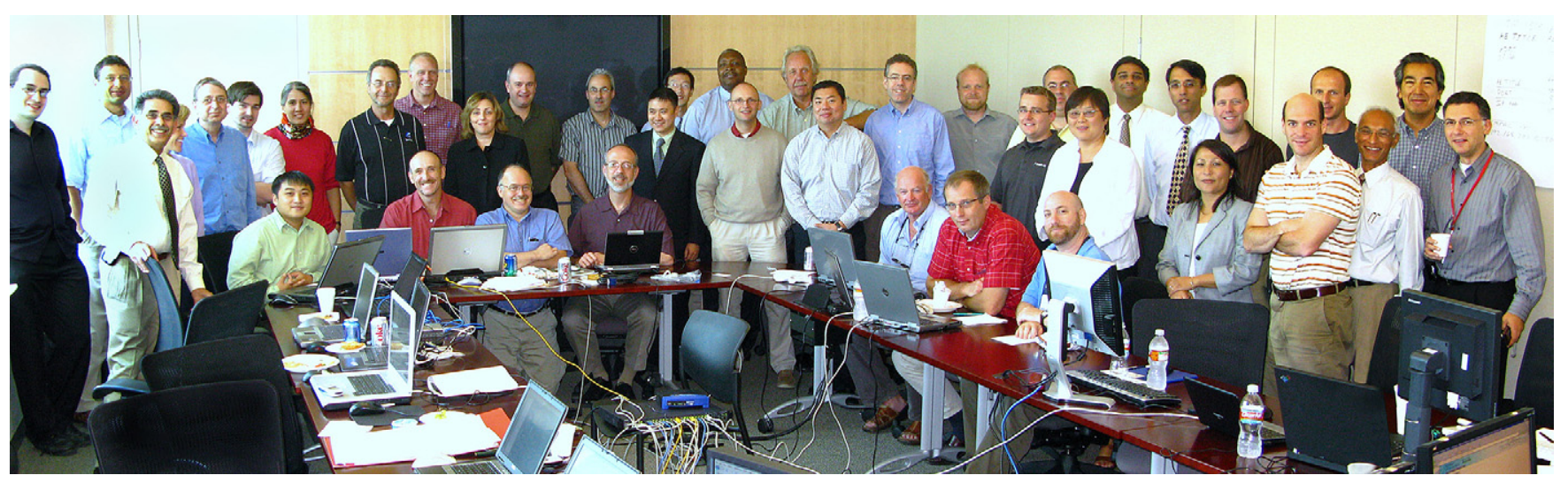

Fig. 2. Participants in the Connectathon held in Houston, TX, Aug 2008. Back row, left to right: Paul Jacobs (MIMVista); Rene Hassanein (Varian Systems); Jatinder Palta, Taskforce Cochair (University of Florida); Jen Anderson (TomoTherapy); Ulrich Busch (Varian Systems); Josef Keller (Siemens); Regula Sie (Varian Systems); Bob Philbin (formerly with TomoTherapy); Virgil Willcut (CMS-Elekta); May Abdel-Wahab, Cochair Planning Committee (University of Miami); Andy Fuller (TomoTherapy); Jaap Pijpelink (Nucletron); Tony Quang (VA Puget Sound/University of Washington); Zhe Chen (Yale-New Haven Hospital); Charles Able (Wake Forest University); Tommy Knoos (ESTRO); Bernd Becker (Siemens); Edward Wu (US Oncology-Cancer Center Services); Richard Poppel (University of Alabama, Birmingham); Mika Miettinen, Cochair Planning Committee (Varian Systems); Christof Schadt (BrainLAB); John Sarafa (CMS); Sha Chang (University of North Carolina); Ramesh Rengan (University of Pennsylvania); Kevin Albuquerque (Loyola University Medical Center), Adam Earwicker (Varian Systems), Sunita Ranjitkar (ASTRO); Christian Sonek (BrainLAB); Mike Bieda (University of Pennsylvania); Prabhakar Tripuraneni, Taskforce Chair (Scripps Clinic); Rick Rocha (Impac); Firas Mourtada (M. D. Anderson Cancer Center). Seated, left to right: Koua Yang (Philips), Mike Courtney (Philips); Bruce Curran, Technical Committee Cochair (Rhode Island Hospital); Walter Bosch (Washington University); Dave Burge (Nucletron); Harold Beunk (Nucletron); Stuart Swerdloff, Technical Committee Cochair (Impac). (Photo courtesy of Prabhakar Tripuraneni, Scripps Clinic, La Jolla, CA.)

been developed through IHE-RO include normal treatment planning, multimodality registration for radiation oncology, and RT treatment workflow. All profiles progress through a series of phases, typically (1) development, (2) public comment, (3) trial implementation, and (4) final text. Figure 1 shows the issues that need to be addressed by the use cases and profiles.

c. Testing at Connectathons. Subsequent face-to-face annual testing to verify seamless connection of the various vendor systems is done. Vendors who pass the test demonstrate their products to the public during the ASTRO annual meeting at the public demonstration described below. Once the integration profiles are finalized, vendors implement these profiles and test their systems with software tools. Successful completion of the testing requires the vendor's system to receive information from at least three other vendors who support the previous steps in the information flow and to transmit information to three vendors whose applications represent the next steps. Figure 2 demonstrates participants at the August 2008 Connectathon in Houston, TX. Vendors who pass the Connectathon (face-to-face testing in front of a panel of judges) for one or more of the profiles are eligible to participate in the IHERO public demonstration that occurs in the exhibit hall during the ASTRO annual meeting. IHE-RO unveiled its first integration profile during its first public demonstration at the 49th Annual ASTRO Meeting in Los Angeles, CA, in 2007.

d. Public demonstrations. Vendors who pass the Connectathon testing for one or more of the profiles are eligible to participate in the IHE-RO public demonstration. During this event, the vendors demonstrate how products are able to seamlessly work with products from other companies. Products are demonstrated to the public in the exhibit hall at the ASTRO annual meeting.

e. Publishing of integration profiles for use in requests for proposals. Incorporation of completed integration profiles into requests for proposals (RFPs) can be done when purchasing new equipment.
Vendors can publish IHE integration statements to document the integration profiles supported by their products. As a result, users will be able to reference integration profiles in requests for proposals, simplifying the systems acquisition process. These IHE-RO solutions are now available in many commercial radiation oncology-related treatment planning, delivery, and information systems. They are also implemented in cancer care sites around the world.

\section{IHE-RO: past, present, and future}

The first interoperability issue identified by IHE-RO from 2005 to 2007 was the inability to support the normal flow of treatment planning data for a simple treatment plan from imaging to dosimetric review. Issues associated with multiple CT series, contouring, and reference point definition, CT-simulation output, and remote viewing of dosimetric data were addressed. The second interoperability issue was the inability to fuse multimodality imaging data with the CT simulation treatment planning data set for contouring. In this case, the use of standardized DICOM objects for sharing registration information across products was addressed, as well as the ability of relevant systems to handle three or more imaging datasets. Integration profiles were developed for each of these issues, test tools were created and made available, and Connectathons were held to validate product implementations. Vendors that successfully incorporated this integration profile were certified and participated in public demonstrations.

\section{DISCUSSION}

Currently (2008-2009), integration profiles are being tested in support of more advanced RT plan exchange (electrons, intensity-modulated RT, and dynamic wedges) and in standardized transfer of imaging and treatment information in support of the radiation oncology delivery process. Ongoing 
efforts are being pursued to develop integration profiles to allow for creation of a composite dose plan consisting of complex treatment planning data from disparate treatment planning systems. Additionally, an integration profile aimed at allowing the transfer of the radiation treatment prescription across treatment planning systems is being developed.

Future use cases in development include the creation of a composite dose plan for proton and photon beam radiotherapy. Additional use cases for integration profiles that will be developed include the integration of bronchoscopy tumor localization data into external beam treatment planning. Efforts are also being pursued to work with the Japanese IHE-RO group to develop mutual use cases for collaborative development of integration profiles.

\section{Benefit to the radiation oncology community}

Radiation oncologists, medical physicists, and administrators can use the resulting technical specifications in RFPs when buying new software/equipment to simplify the buying process and lead to better connectivity and smoother integration of the new equipment with the existing ones in the department. The process also helps enhance vendor efficiency by highlighting issues with connectivity early in the development phase of the product.

Healthcare professionals are encouraged to request and use IHE-RO-compatible products and increase their awareness of IHE-RO activities in the radiation oncology community, use IHE-RO integration profiles in their RFPs for their new products, and recommend their vendors to participate in IHE-RO. For more information visit http://wiki.ihe.net.

\section{CONCLUSIONS}

IHE-RO efforts develop integration profiles that can be incorporated into RFPs for new products. This can enhance connectivity and integration of new equipment in radiation oncology departments.

\section{REFERENCE}

1. Swerdloff SJ. Data handling in radiation therapy in the age of image-guided radiation therapy. Semin Radiat Oncol 2007;17(4): 287-292. 